\title{
MEASUREMENTS OF COUPLING IMPEDANCE FOR THE SNS ACCUMULATOR RING
}

\author{
J. G. Wang, ORNL, Oak Ridge, TN 37831, USA \\ S. Y. Zhang, BNL, Upton, NY 11973, USA
}

\begin{abstract}
The measurements of coupling impedance for the US Spallation Neutron Source (SNS) ring are presented. The work involves a strip-line Beam Position Monitor (BPM) and a window-frame kicker magnet. The conventional wire method is employed with improved formulas.
\end{abstract}

\section{INTRODUCTION}

The SNS Accumulator Ring is designed to contain a proton bunch of $2 * 10^{14}$ particles at $1 \mathrm{GeV}$ with a repetition rate of $60 \mathrm{~Hz}$ for achieving $2 \mathrm{MW}$ output power. This will be the highest intensity of proton beams in the world. Care must be taken to prevent collective instabilities. An accurate impedance budget of the ring components supported by experiments is essential. Therefore, an important piece of the ring design is to measure the coupling impedance of some critical ring components and to compare with theory, model and simulation in order to gain confidence in the ring design. In this paper we report the measurements of coupling impedance of a strip-line BPM and a window-frame kicker magnet for the SNS accumulator ring [1].

\section{WIRE METHOD}

The conventional wire-method [2-6] is employed in the experiment. For the measurement of longitudinal coupling impedance a thin metallic wire is installed on the axis of the Device Under Test (DUT) and a reference pipe (REF) to form coaxial transmission line configurations. The scattering coefficients $S_{21}$ and $S_{11}$ are obtained with a network analyser, which should be matched to the DUT/REF. The measurement of the transverse coupling impedance requires a dipole current moment which can be generated with two parallel wires installed in the DUT/REF. Following V.G. Vaccaro's approach [7], we have developed new formulas for coupling impedance calculations [8]:

$$
Z_{/ /} \cong 2 Z_{0} \operatorname{In}\left(\frac{S_{c}^{R}}{S_{c}^{D}}\right),
$$

and

$$
Z_{\perp} \cong \frac{c}{\omega \Delta^{2}} 2 Z_{0} \ln \left(\frac{S_{c}^{R}}{S_{r}^{D}}\right) \text {. }
$$

Here $Z_{0}$ is the characteristic impedance formed by the REF/DUT with the central wires, $\Delta$ the separation of the two wires in the transverse impedance measurement, the superscripts R and D for the REF and DUT, respectively, and $S_{c}$ is a corrected scattering coefficient obtained from

$$
S_{c}^{2}+\frac{S_{11}^{2}-S_{21}^{2}-1}{S_{21}}+1=0 \text {. }
$$

\section{MEASUREMENT OF A BPM}

The coupling impedance of a strip-line BPM is well predicted by the transmission line theory. The measurement of such a device would allow us to validate the method of our experiment. Based on the ideal transmission line theory, the longitudinal coupling impedance of a strip-line BPM can be calculated as [9]

$$
\begin{aligned}
& Z_{r}=N Z_{c}\left(\frac{\phi_{0}}{2 \pi}\right)^{2} \operatorname{Sin}^{2}\left(\frac{\omega l}{c}\right), \\
& Z_{i}=N Z_{c}\left(\frac{\phi_{0}}{2 \pi}\right)^{2} \operatorname{Sin}\left(\frac{\omega l}{c}\right) \operatorname{Cos}\left(\frac{\omega l}{c}\right) .
\end{aligned}
$$

Here $N$ is the number of strip-line probes in a BPM, $Z_{c}$ is the characteristic impedance of the strip line, $\phi_{0}$ is the angle subtended by the probe, $l$ is the length of the strip line, and $\omega$ is the signal frequency. The strip-line BPM in the measurement, i.e. the DUT, contains two probes, each of which has a characteristic impedance of $50 \Omega$, a length of $0.275 \mathrm{~m}$, a subtended angle $\phi_{0}$ of 80 degree. In order to compare with the theoretical result from Eq. (4), the REF used in the measurement is the same mechanical structure as the DUT, except that the two probes are taken out.

The longitudinal coupling impedance of the strip-line BPM from the measurement is shown in Fig. 1, where both the real and imaginary parts are plotted as a function of frequency. The theoretical result from Eq. (4) is also depicted for comparison with the measurement. The agreement is reasonably good. The discrepancy is first attributed to the coupling between the two probes, which is not included in Eq. (4). Other factors of causing the discrepancy include the end effect of the real transmission-line probes and distributed parameters, especially at higher frequencies. 


\section{MEASUREMENT OF A KICKER}

The design of the SNS extraction kicker is very similar to the Booster dump kicker at BNL in that they use the same window frame ferrite magnets. The difference is in that there are two sections in the Booster dump kicker, while the SNS will use as many as 14 sections. The coupling impedance of the extraction kicker is probably the largest among all the SNS ring components, that raises some concerns about possible instabilities of beams. A measurement of the Booster dump kicker should provide useful information on the design of the SNS extraction kicker.

The Booster dump kicker (DUT) contains two sections of window frame ferritc magnets, cach of which has a dimension of 4.65" (height) x 6.23" (width) $\times 16$ " (length), and a ferrite thickness of 1". A copper sheet break of $1 \mathrm{~mm}$ in thickness is inserted in the top, as well as the bottom, of the magnet to reduce beam power loss. A smooth beam pipe with the same length as the DUT's is used as the reference (REF).

For the longitudinal coupling impedance measurement, a thin central wire is installed on the axis of the REF/DUT, which forms a coaxial transmission line with characteristic impedance of about $370 \Omega$. Matching $50 \Omega$ of the network analyser to $370 \Omega$ of the reference line is achieved by adding resistive networks.

The longitudinal impedance of the kicker magnets from the measurement is shown in Fig. 2. The real part of the impedance has a peak value of about $110 \Omega$ around 25 $\mathrm{MHz}$, and then decreases monotonously when the frequency increases. The imaginary part of the impedance is inductive within the test frequency range. The measurement also shows that the longitudinal coupling impedance of the window frame magnet is not sensitive to external loads.

The measurement of the transverse coupling impedance requires a dipole current moment, which can be generated with two parallel wires. By employing wide-band transformers, the common mode signal from a network analyzer can be converted to differential mode signals inside the DUT or REF. Matching is still to be made, e.g., by resistive networks. The scattering coefficients are then obtained from the network analyzer for impedance calculation.

In Fig. 3 (a) and (b), we plot the real and imaginary part of the transverse coupling impedance of the dump-kicker magnet with different loads. It can be seen that the result is very sensitive to the external loads, in contrast to the longitudinal case.

When a $18 \mathrm{~m}$ long, open-end $50 \Omega$ cable is connected to the magnet power supply terminals, its transverse coupling impedance shows resonant structures, which is potted in Fig. 4.

\section{SUMMARY}

The coupling impedance of a strip-line BPM and a Booster dump-kicker magnet has been measured for the SNS project. The conventional wire method is employed for the bench test, with newly derived formulas to calculate the coupling impedance from experimental data. For the strip-line BPM, the longitudinal coupling impedance has been measured and compared with theory. The agreement is reasonably good, that validates our experimental method. For the dump-kicker magnet, both the longitudinal and transverse impedance has been measured. In comparison with the models, which are not shown in this paper, the measurement has provided useful information about the parasitic power loss in the magnet, which should be investigated further. Both the longitudinal and transverse impedance of the dump-kicker magnet appears to be very high. This has raised some concerns about 14 sections of the extraction kicker magnets for the SNS ring, which may lead to instabilities. Our experience also suggests that the coupling impedance of other critical components such as the RF cavity, collimators on the SNS ring should also be measured before the ring design is finalized.

\section{ACKNOWLEDGEMENTS}

We would like to acknowledge the support of many collcagucs in the BNL/SNS team. Especially, the first author is indebted to R. Sikora and J. Cupolo for their very helpful assistance in mechanical and electronics work of the experiment. Useful discussions with H. Hahn on the BPM coupling impedance measurement are also acknowledged.

\section{REFERENCES}

[1] J.G. Wang and S.Y. Zhang, BNL/SNS Technical Note No. 079, June 2000.

[2] A. Faltens et al, $8^{\text {th }}$ Int. Conf. on high-energy accelerators, CERN, 1971, p. 338.

[3] M. Sands and J. Rees, SLAC-Report PEP-95, August 1974.

[4] H. Hahn and F. Pedersen, BNL-Report 50870, in Particle Accelerators and High-Voltage Machines TID-4500, April 1978.

[5] G. Nassibian and F. Sacherer, in NIM 159 (1979), p. 21.

[6] L.S. Walling et al, in NIM, A281 (1989), p. 433.

[7] V. G. Vaccaro, INFN/TC-94/023, Sezione di Napoli, November 1994.

[8] J. G. Wang and S.Y. Zhang, submitted to NIM for publication.

[9] K. Y. Ng, AIP Conf. Proc. 184, p. 499, 1989. 


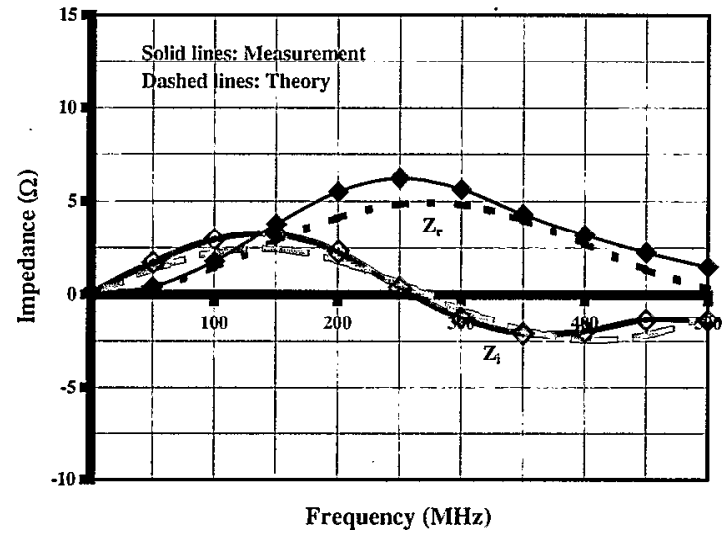

Fig. 1 Longitudinal coupling impedance of the BPM.

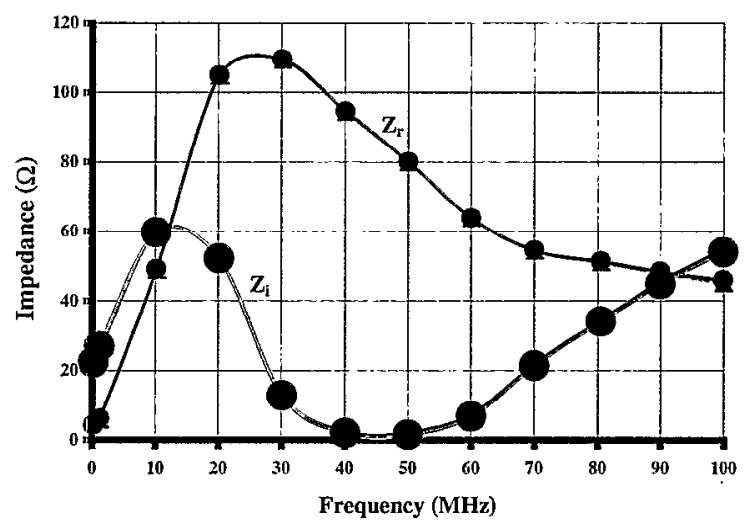

Fig. 2 Longitudinal impedance of the kicker.

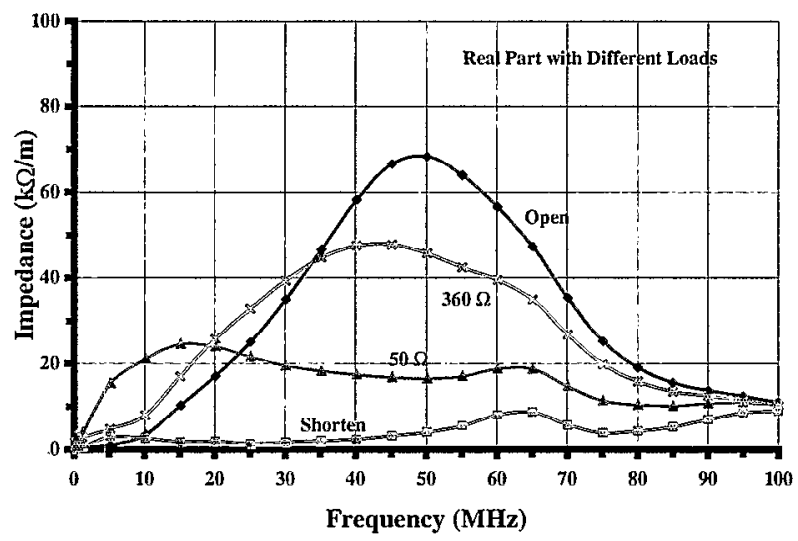

Fig. 3(a) Measured transverse impedance of the dump kicker with different loads: real part.

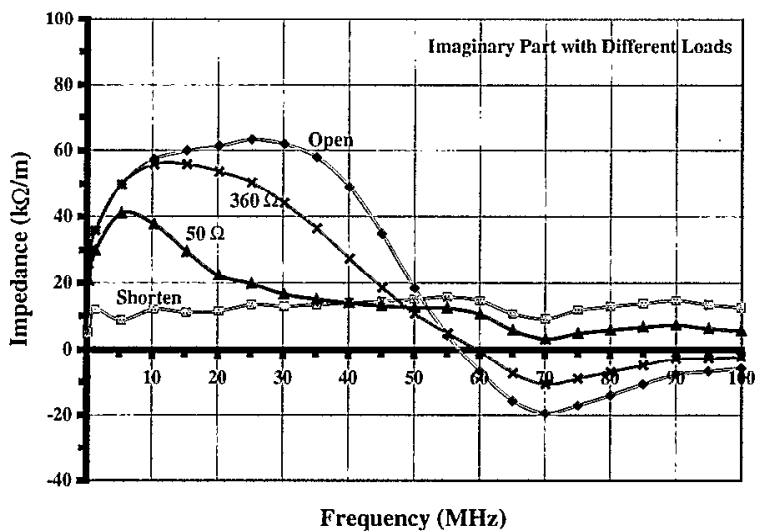

Fig. 3(b) Measured transverse impedance of the dump kicker with different loads: imaginary part.

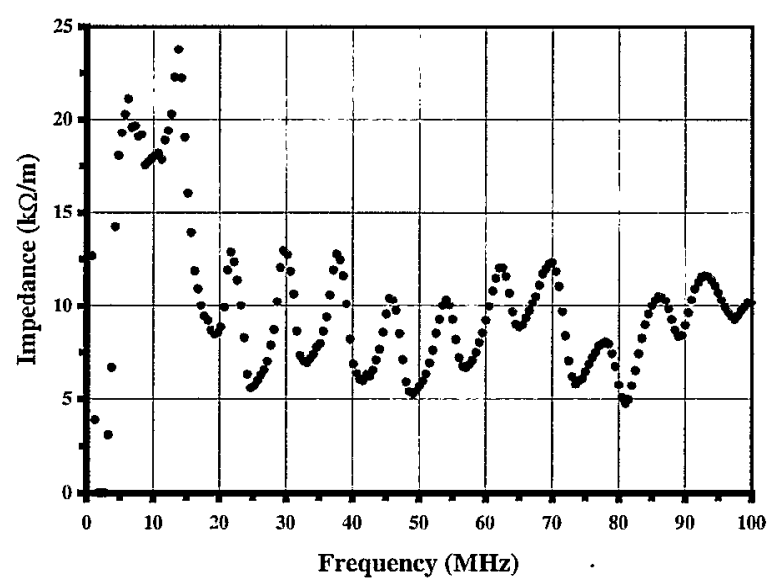

Fig. 4 Transverse impedance of the dump kicker with an open $50 \Omega$ cable as the load. 